\title{
An Integrated-Plasmonic Chip of Bragg Reflection and Mach-Zehnder Interference Based on Metal-Insulator-Metal Waveguide
}

\author{
Liang ZENG ${ }^{1}$, Jiaqi $\mathrm{LI}^{1}$, Chengyi CAO ${ }^{1}$, Xudong $\mathrm{LI}^{1}$, Xinyuan ZENG ${ }^{1}$, Quanhao YU ${ }^{1}$, \\ and Kunhua WEN ${ }^{1,3^{*}}$, Jun YANG ${ }^{2,3}$, and Yuwen QIN ${ }^{2,3}$ \\ ${ }^{1}$ School of Physics and Optoelectronic Engineering, Guangdong University of Technology, Guangzhou 510006, China \\ ${ }^{2}$ School of Information Engineering, Guangdong University of Technology, Guangzhou 510006, China \\ ${ }^{3}$ Guangdong Provincial Key Laboratory of Information Photonics Technology, Guangdong University of Technology, \\ Guangzhou 510006, China
}

${ }^{*}$ Corresponding author: Kunhua WEN_Ｅ-mail: khwen@gdut.edu.cn

\begin{abstract}
In this paper, a Bragg reflector is proposed by placing periodic metallic gratings in the center of a metal-insulator-metal (MIM) waveguide. According to the effective refractive index modulation caused by different waveguide widths in a period, a reflection channel with a large bandwidth is firstly achieved. Besides, the Mach-Zehnder interference (MZI) effect arises by shifting the gratings away from the waveguide center. Owing to different optical paths with unequal indices on both sides of the grating, a narrow MZI band gap will be obtained. It is interesting to find out that the Bragg reflector and Mach-Zehnder interferometer are immune to each other, and their wavelengths can be manipulated by the period and the grating length, respectively. Additionally, we can obtain three MZI channels and one Bragg reflection channel by integrating three different gratings into a large period. The performances are investigated by finite-difference time-domain (FDTD) simulations. In the index range of $1.33-1.36$, the maximum sensitivity for the structure is as high as $1500 \mathrm{~nm} / \mathrm{RIU}$, and it is believed that this proposed structure can find widely applications in the chip-scale optical communication and sensing areas.
\end{abstract}

Keywords: Bragg reflector; metal-insulator-metal; Mach-Zehnder interference; effective refractive index modulation

Citation: Liang ZENG, Jiaqi LI, Chengyi CAO, Xudong LI, Xinyuan ZENG, Quanhao YU, et al., "An Integrated-Plasmonic Chip of Bragg Reflection and Mach-Zehnder Interference Based on Metal-Insulator-Metal Waveguide," Photonic Sensors, 2022, 12(3): 220303.

\section{Introduction}

Surface plasmon polaritons (SPPs) are collective oscillations generated by the interaction between the electromagnetic waves and the free electrons on the metal surface [1]. Since SPPs can break through optical diffraction limit, they bring the possibility of integration of photonic circuits and electronic circuits. As one of the most popular plasmonic structures that manipulate light at the sub-wavelength scale, the metal-insulator-metal (MIM) waveguide has been widely concerned for its advantages of the long transmission distance, precise light field control, and easy fabrication [2-11].

Received: 19 July 2021 / Revised: 30 November 2021

(C) The Author(s) 2022. This article is published with open access at Springerlink.com

DOI: $10.1007 / \mathrm{s} 13320-022-0650-0$

Article type: Regular 
Consequently, more and more nano-scale optical devices based on MIM waveguides have been proposed and investigated, such as the optical demultiplexer, power splitter, wavelength filter, optical switch [12-27]. It is no doubt that one would like to integrate those important functional devices into a chip for realizing photonic circuits and electronic circuits. For instance, fiber Bragg gratings (FBGs) and Mach-Zehnder interferometers (MZIs) are widely used in the optical fiber communication and sensing systems [28-33]. Therefore, it would be interesting to produce their performances in the chip-scale circuits rather than in the traditional fiber waveguides [34, 35].

In this paper, an MIM waveguide with periodic metallic gratings (PMGs) that are used to modulate the refractive index (RI) is proposed. When the PMGs are laid in the center of the waveguide, broadband Bragg reflection is achieved owing to the periodic RI modulation. In addition, MZI performance is also obtained when the PMGs are moved away from the center, for the reason that two asymmetrical waveguide widths lead to slightly index differences of two optical paths. By cascading different MZI arms, up to three interference band gaps and one Bragg reflection channel are achieved in the proposed structure. The parameters of the proposed structure are fully considered to investigate the performances by using the finitedifference time-domain (FDTD) method.

\section{Performance analysis}

Figure 1(a), which shows the three-dimensional schematic diagram of the plasmonic MIM device, provides an intuitive understanding of the whole structure. Since oxidation will affect the performance, the metallic structure is deposited on a thin silica layer to avoid the oxidation. The two-dimensional structure is shown in Fig. 1(b), and it is employed in the FDTD simulation for saving running time and hardware resources. The width of the bus MIM waveguide is defined as $W$. The length, thickness, and period of the PMG are $L_{a}, d$, and $P$, respectively. The distances from the metallic grating to the upper and lower edges of the MIM waveguide are $L_{u}$ and $L_{d}$, respectively. It means that we can obtain the equivalent widths for the two optical paths on both sides of the grating. According to the dispersion characteristics of the TM mode in the MIM waveguide [36], the relationship between the effective RI of $n_{\text {eff }}$ and other parameters, such as the dielectric constants of materials and the waveguide width, can be expressed as

$$
\begin{gathered}
\varepsilon_{i} k_{m}+\varepsilon_{m} k_{i} \tanh \left(\frac{-\mathrm{j} k_{i} w}{2}\right)=0 \\
k_{i, m}=\sqrt{\varepsilon_{i, m} k_{o}^{2}-\beta^{2}} \\
n_{\text {eff }}=\frac{\beta}{k_{0}}
\end{gathered}
$$

where $\varepsilon_{i, m}$ and $k_{i, m}$ are the dielectric constants and the transverse propagation constants of the insulator and metal, respectively, $\beta$ and $k_{0}$ are the propagation constants of the waveguide and vacuum, respectively, and $w$ is the width of the waveguide. It should be noted here that the phase modulation is determined by the corresponding real part $\operatorname{Re}\left(n_{\text {eff }}\right)$. The propagation loss, which may affect the transmittance and the full width at half maximum (FWHM), is mainly determined by the image part of the effective index, and it can also be obtained by (1) - (3). However, in this proposed structure, we mainly focus on the wavelengths of the MZI and Bragg reflection effects determined by $\operatorname{Re}\left(n_{\text {eff }}\right)$, so the transmission loss is not currently considered in the following theoretical analysis model, but will be covered in all the FDTD simulations. In the following, we firstly assume the metal and insulator as silver and air, respectively. The relative permittivity of silver is characterized by the Drude model: $\varepsilon_{m}=\varepsilon_{\infty}-\omega_{p}^{2} /\left(\omega^{2}+\mathrm{i} \omega \gamma\right)$ with $\varepsilon_{\infty}=3.7$, $\omega_{p}=9.1 \mathrm{eV}$, and $\gamma=0.018 \mathrm{eV}[37,38]$. In this case, the values of $\operatorname{Re}\left(n_{\text {eff }}\right)$ can be calculated according to (1) - (3), as shown in Fig. 2(a) if we 
define the waveguide width as $w=50 \mathrm{~nm}, 60 \mathrm{~nm}$,

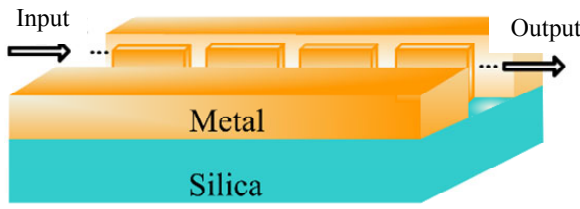

(a)
$70 \mathrm{~nm}$, and $150 \mathrm{~nm}$, respectively.

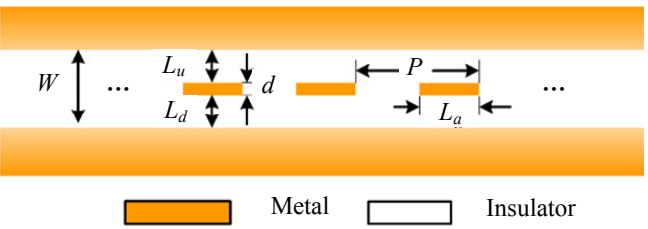

(b)

Fig. 1 Schematic diagram of the plasmonic MIM structure: (a) three-dimensional view and (b) corresponding two-dimensional view.

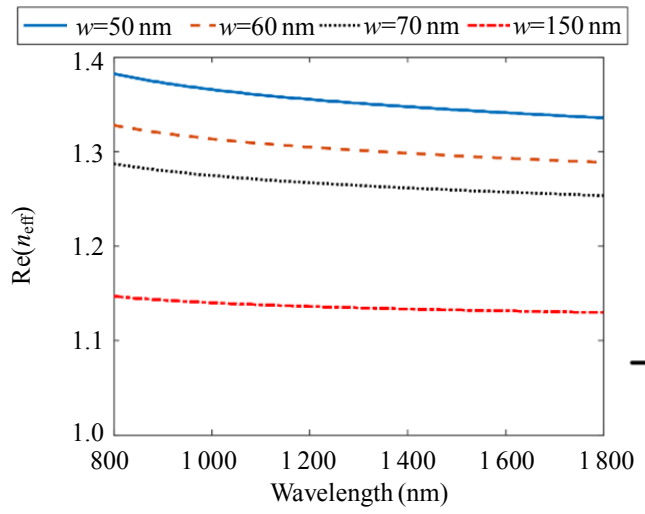

(a)

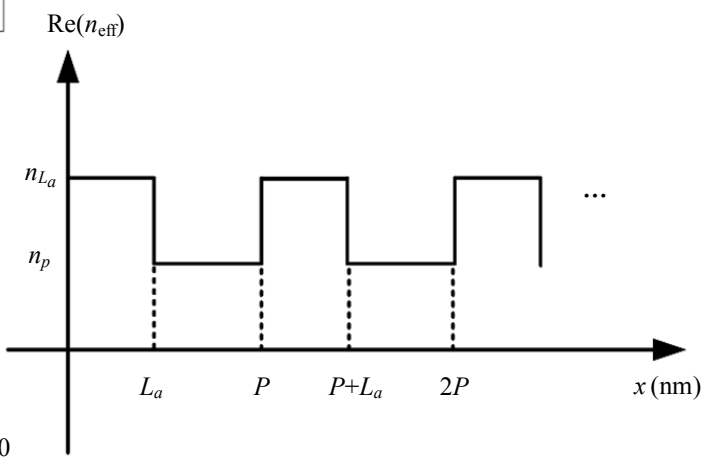

(b)

Fig. 2 Relationship between $\operatorname{Re}\left(n_{\text {eff }}\right)$ and the wavelength or the waveguide: (a) $\operatorname{Re}\left(n_{\text {eff }}\right)$ with respect to the wavelength by defining the waveguide width as $W=50 \mathrm{~nm}, 60 \mathrm{~nm}, 70 \mathrm{~nm}$, and $150 \mathrm{~nm}$, respectively and (b) variation of $\operatorname{Re}\left(n_{\text {eff }}\right)$ along with the MIM waveguide.

Obviously, $\operatorname{Re}\left(n_{\text {eff }}\right)$ will increase as the waveguide width decreases, and it will also decrease with an increase in the wavelength. In the following FDTD simulation, the width of the bus MIM waveguide is fixed as $W=150 \mathrm{~nm}$ through the paper. The grating thickness is defined as $d=30 \mathrm{~nm}$, the distances are $L_{u}=L_{d}=(W-d) / 2=60 \mathrm{~nm}$, the length is $L_{a}=240 \mathrm{~nm}$, and the grating period is $P=645 \mathrm{~nm}$. It means that the length of the empty section in a period is $P-L_{a}=645-240=405 \mathrm{~nm}$. Because the grating is symmetrically laid in the center of the MIM waveguide, there is no index difference and additional phase delays for the two optical paths on both sides of the grating, which means that no interference occurs and they can be equivalent to one path with a width of $60 \mathrm{~nm}$. In this case, the index modulation for the whole structure is shown in Fig. 2(b), which illustrates the periodic variation of index along with the waveguide. According to the results in Fig. 2(a), the values of $n_{L}$ and $n_{P}$ within a grating period are about 1.287 and 1.134 at the wavelength of $1550 \mathrm{~nm}$, respectively. In particular, the widths used for calculating $n_{p}$ and $n_{L_{a}}$ are $W=$ $150 \mathrm{~nm}$ and $L_{u}=L_{d}=60 \mathrm{~nm}$, respectively, as shown in Fig. 1(b). The effective index is only determined by the width of the waveguide and will not be affected by the length. It should be mentioned that the oxygen plasma etching technology and magnetron sputtering technology have been applied to fabricate such kind of the MIM waveguide, and the processing capacity is up to $10 \mathrm{~nm}$ and $3.3 \mathrm{~nm}$ $[39,40]$, respectively. Although there is no problem for the reproducibility of the proposed system based on the current technologies, one should also concentrate on the processing accuracy which will affect the performance of the device.

According to the Bragg condition, we can derive the central wavelength $\lambda_{B}$ of the forbidden band as

$$
\lambda_{B}=2 n_{B} P
$$




$$
n_{B}=\left(n_{\mathrm{L}_{\mathrm{a}}}+n_{P}\right) / 2
$$

where $n_{B}$ is the equivalent refractive index of the grating, and the denominator " 2 " is a fitting number. The 2-dimentional (2D) FDTD method is employed to investigate the transmission responses of this proposed structure. It is no doubt that the simulation accuracy of the 3-dimentional (3D) model is more accurate than that of the $2 \mathrm{D}$ model. But for the integrated optical components, 2D simulations have been widely used even with a little penalty of accuracy $[6-8,12-14,17,18,20,21]$, but the hardware resources and running time can be reduced. The perfectly matched layers have been used as the boundary conditions during the FDTD simulations. By setting the total periods as 15 , the reflection and transmission spectra based on the FDTD simulation are shown in Fig. 3 with a red-dash line and a black-solid line, respectively. In the spectrum, there is a broadband reflection peak at the wavelength of $1550 \mathrm{~nm}$, which agrees well with the analysis result in (4) with $n_{B}=1.2106$. The FWHM is about $170 \mathrm{~nm}$ and the maximum reflection is about 0.86 , which provide the possibility of broadband filtering in the optical communication area. Figures 4(a) and 4(b) are the magnetic field distributions of the structure at the high-transmission wavelength of $1440 \mathrm{~nm}$ and at the gap wavelength of $1550 \mathrm{~nm}$, respectively. They can further investigate the SPP propagation details inside the MIM waveguide. Specifically, strong field energy is distributed within the whole waveguide in Fig. 4(a), while the field becomes weaker and weaker along with the waveguide owing to the Bragg reflection.

Moreover, other parameters that will significantly affect the grating performances are analyzed in Fig. 5, including the period $P$, the length $L_{a}$, and the thickness $d$. When one of the parameters is changed, the others remain unchanged and are the same as that in Fig. 2. Firstly, we define $P=400 \mathrm{~nm}$, $500 \mathrm{~nm}$, and $600 \mathrm{~nm}$ in Fig. 5(a) to illustrate the transmission spectra. Based on (4), it is known that the peak wavelength has a linear relationship with

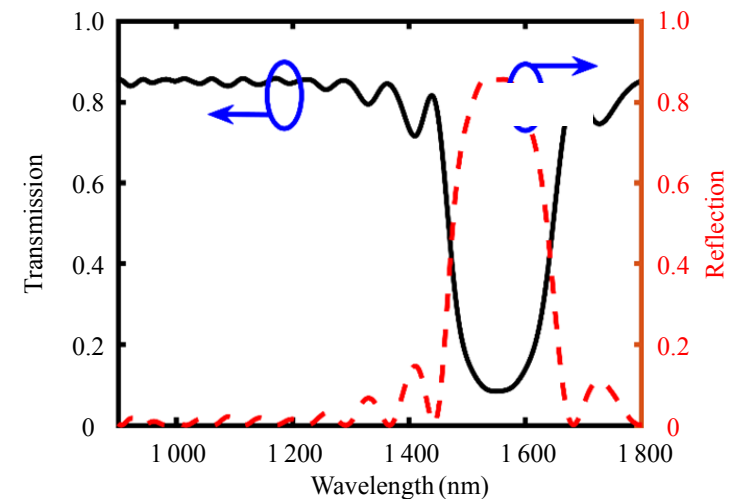

Fig. 3 Reflection spectrum (red-dash line) and transmission spectrum (black-solid line) with $P=645 \mathrm{~nm}$.

$P$, and it is also an important factor determining the Bragg wavelength. Consequently, the peak wavelengths of $994 \mathrm{~nm}, 1220 \mathrm{~nm}$, and $1450 \mathrm{~nm}$ in Fig. 5(a) have a variation ratio that is in accordance with the ratio of $P$. One can flexibly design the period of the structure for obtaining the expected wavelength. In Fig. 5(b), the length of the grating in a period is defined as $L_{a}=200 \mathrm{~nm}, 240 \mathrm{~nm}$, and $280 \mathrm{~nm}$, respectively. In this situation, it is interesting to find out that the transmission spectrum has only a little red shift, i.e., $1212 \mathrm{~nm}, 1220 \mathrm{~nm}$, and $1236 \mathrm{~nm}$, respectively. This characteristic provides the possibility of integrating MZI performance by changing $L_{a}$ while keeping the Bragg reflection band. Finally, the thickness $d$ of the metallic grating is defined as $30 \mathrm{~nm}, 40 \mathrm{~nm}$, and $50 \mathrm{~nm}$, i.e., $L_{a}=L_{u}=60 \mathrm{~nm}, 55 \mathrm{~nm}$, and $50 \mathrm{~nm}$, respectively. An increase in $d$ means that the widths of waveguides on both sides of the grating will decrease, leading to an increase in $n_{L_{a}}$ based on (4). According to the grating theory, the modulation depth $\Delta n=n_{L_{a}}-n_{P}$ is enhanced because $n_{P}$ is uniform in these cases. Therefore, the FWHM becomes larger and the transmission is lower, leading to a flat bottom of the band in Fig. 5(c).

Furthermore, we are going to analyze the situation that the metallic grating is shifted $10 \mathrm{~nm}$ away from the center of the MIM waveguide. It means that $L_{u}=50 \mathrm{~nm}$ and $L_{d}=70 \mathrm{~nm}$ result in different indices of $n_{L_{u}}$ and $n_{L_{d}}$, as well as 
different phase delays for the optical paths on both sides of the grating. According to the MZI theory and the proposed structure, the criterion of the MZI condition can be expressed as

$$
\frac{2 \pi\left(n_{L_{u}}-n_{L_{d}}\right) L_{a}}{\lambda}=(2 m+1) \pi
$$

where $\lambda$ represents the wavelength of the $m$ th valley, and $m$ is an integer.

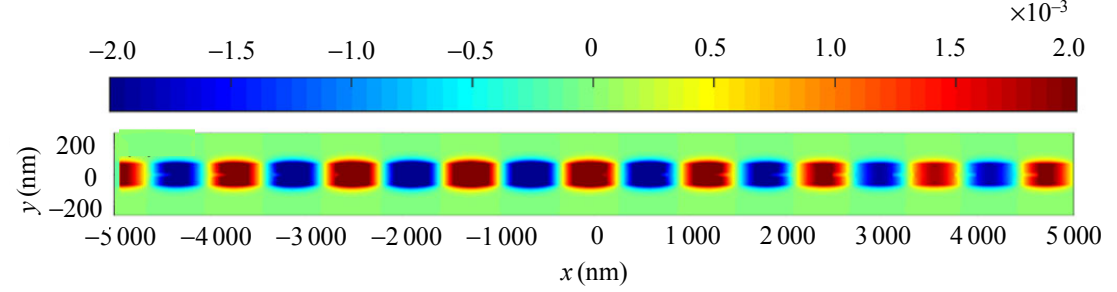

(a)

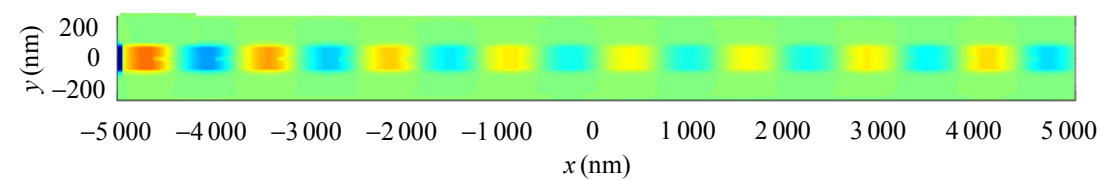

(b)

Fig. 4 Magnetic field distributions of the grating at (a) $1440 \mathrm{~nm}$ and (b) $1550 \mathrm{~nm}$.

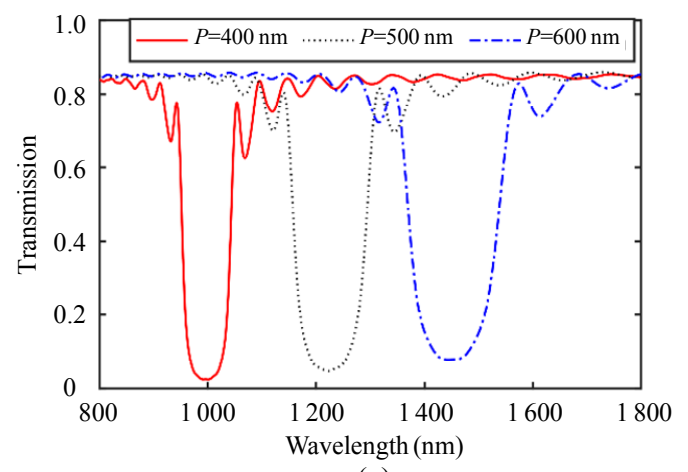

(a)

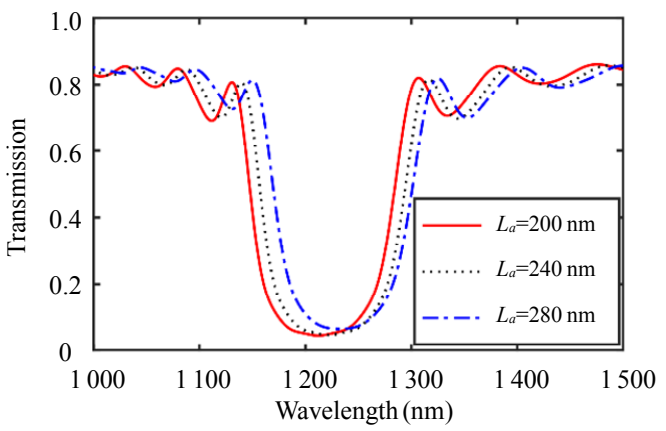

(b)

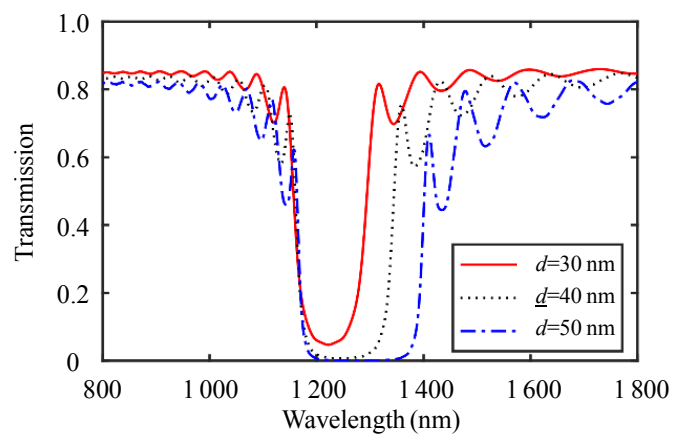

(c)

Fig. 5 Transmission spectra: (a) different grating periods of $400 \mathrm{~nm}, 500 \mathrm{~nm}$, and $600 \mathrm{~nm}$, respectively, (b) different lengths of metal gratings of $200 \mathrm{~nm}, 240 \mathrm{~nm}$, and $280 \mathrm{~nm}$, respectively, and (c) different widths of metal gratings of $30 \mathrm{~nm}, 40 \mathrm{~nm}$, and $50 \mathrm{~nm}$, respectively.

As a result, the MZI effect will occur at the end of each metallic grating, where is also the wider part of a period. Here, it should be pointed out that the Bragg reflection still works in this case, although $n_{L_{a}}$ of the waveguides on both sides of the grating are a little different. After setting $L_{a}=200 \mathrm{~nm}$ and
$P=500 \mathrm{~nm}$ firstly, the FDTD simulation result is shown in Fig. 6(a) with a black-solid line. It can be seen that in addition to the Bragg reflection band at $1214 \mathrm{~nm}$ with FWHM $=76 \mathrm{~nm}$, there is another narrow forbidden band emerging at $815 \mathrm{~nm}$ with $\mathrm{FWHM}=2 \mathrm{~nm}$ because of the MZI effect. 


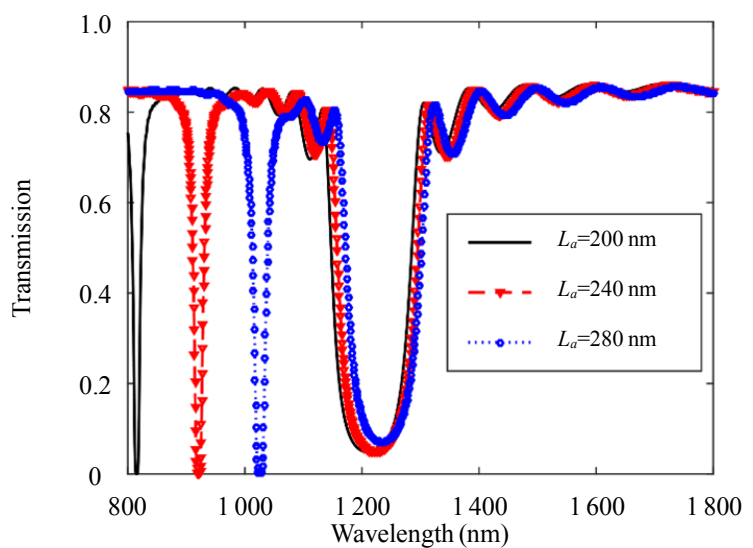

(a)

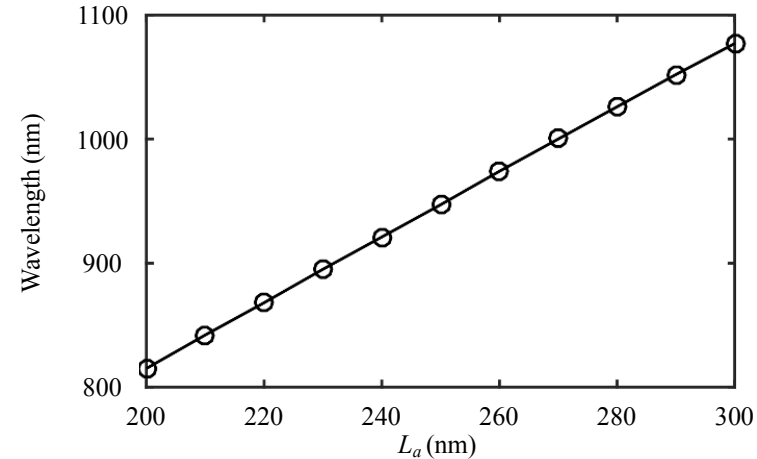

(b)

Fig. 6 Transmission spectra and MZI wavelength under different conditions: (a) transmission spectra by setting $L_{u}=50 \mathrm{~nm}, L_{d}=$ $70 \mathrm{~nm}$ and $L_{a}=200 \mathrm{~nm}, 240 \mathrm{~nm}$, and $280 \mathrm{~nm}$, respectively and (b) MZI wavelength with respect to the variation of $L_{a}$.

Aside from the index modulation, the lengths of both arms in the MZ interferometer will also affect the wavelength. Consequently, the length of $L_{a}$ is changed to be $240 \mathrm{~nm}$ and $280 \mathrm{~nm}$ in Fig. 6(a), and the spectra are plotted with a blue-triangle line, and a red-circle line, respectively. It is interesting to see that the Bragg reflection bands still remain at $\sim 1214 \mathrm{~nm}$ with similar performances. The results further confirm that the shift of the metallic grating will not remove the Bragg reflection effect and its length has only a little influence on the spectrum. However, the MZI response will be greatly affected by the grating length, as the central wavelengths are changed to be $921 \mathrm{~nm}$ and $1026 \mathrm{~nm}$ after increasing $L_{a}$ from $240 \mathrm{~nm}$ to $280 \mathrm{~nm}$. More details can be figured out in Fig. 6(b) by setting $L_{a}$ from $200 \mathrm{~nm}$ to $300 \mathrm{~nm}$ with a step of $10 \mathrm{~nm}$. The MZI wavelengths are increased from $815 \mathrm{~nm}$ to $1077 \mathrm{~nm}$ with a uniform step of $\sim 26 \mathrm{~nm}$. Therefore, it can be concluded that the MZI wavelength is changed linearly with $L_{a}$ in this proposed structure. However, $L_{a}$ should not be excessively enlarged to avoid affecting the performance in this structure, since the channels attributed by the Bragg reflection and interference will be overlapped.

In addition, the field distributions at the wavelengths of the MZI and Bragg reflection are shown in Figs. 7 (a) and 7(b), respectively. Firstly, the field distribution at $921 \mathrm{~nm}$, corresponding to the
MZI dip in Fig. 6(a) with $L_{a}=240 \mathrm{~nm}$, obviously shows that the fields on both sides of the grating are with antiphase. It means that destructive interference will occur at the end of the grating after shifting the grating away from the center. Similar performance has also been investigated in the symmetry-broken metamaterial absorbers [41]. However, the field distribution at $1224 \mathrm{~nm}$ caused by the Bragg reflection shows that the SPP phases on both sides of the grating are identical in Fig. 7(b). SPPs are gradually reflected back to the input port, resulting in a low transmission at the output port. Consequently, the details of the field distributions at the dips caused by the MZI and Bragg reflection are different.

Furthermore, we cascade three small and uniform grating periods into a large period with a length of $P_{i}$ to obtain more MZI channels, as shown in Fig. 8(a). $L_{a 1}, L_{a 2}$, and $L_{a 3}$ are $200 \mathrm{~nm}, 240 \mathrm{~nm}$, and $280 \mathrm{~nm}$, respectively, while $P$ is $500 \mathrm{~nm}$. The FDTD simulated spectrum in Fig. 8(b) investigates that three MZI channels are achieved and their central wavelengths are almost the same as those in Fig. 6(a), respectively. Moreover, the Bragg reflection band named B locates at $1224 \mathrm{~nm}$. Three MZI channels named M1, M2, and M3 are achieved at $815 \mathrm{~nm}, 923 \mathrm{~nm}$, and $1030 \mathrm{~nm}$, respectively. Consequently, one can enhance the integration by cascading different gratings with specific lengths of 
$L_{a}$. The wavelengths of the Bragg reflector and the MZI channels can be adjusted by $P$ and $L_{a}$, respectively.

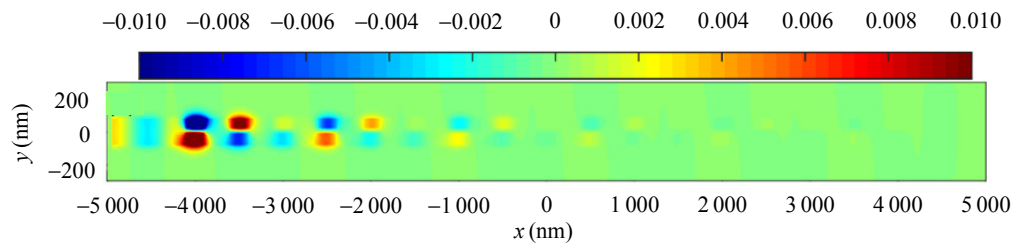

(a)

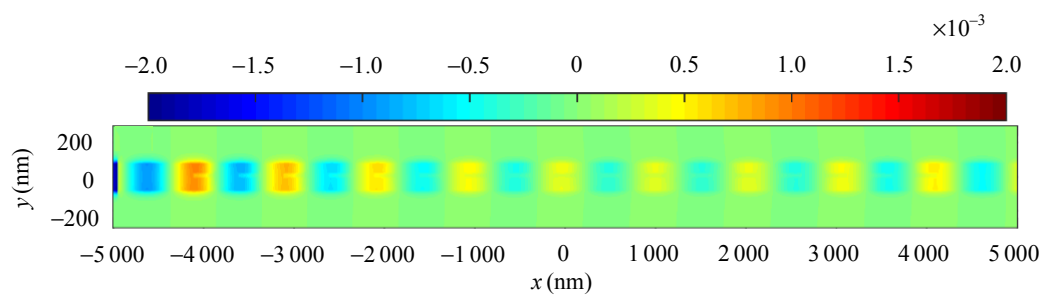

(b)

Fig. 7 Field distributions corresponding to Fig. 6(a) with $L_{a}=240 \mathrm{~nm}$ and $P=500 \mathrm{~nm}$ : (a) MZI dip of $921 \mathrm{~nm}$ and (b) Bragg reflection dip of $1224 \mathrm{~nm}$.

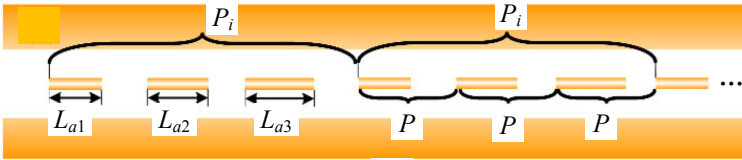

(a)

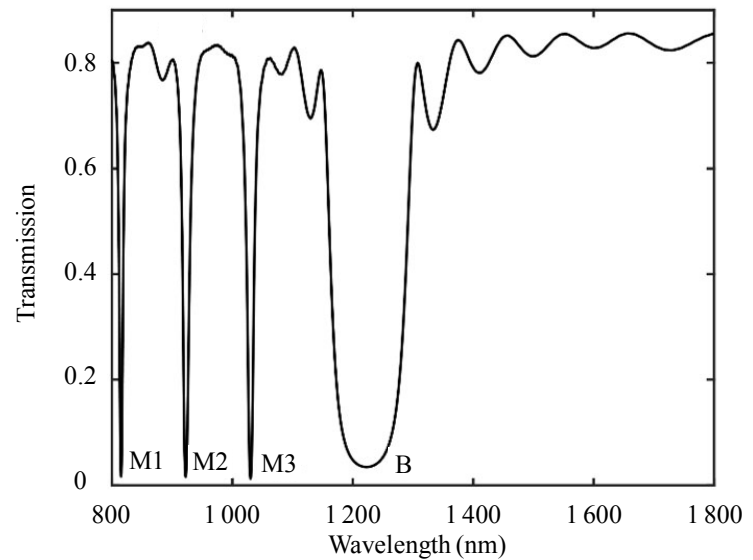

(b)

Fig. 8 Schematic diagram and corresponding spectrum: (a) schematic diagram of three gratings integrated in a large period and (b) transmission spectrum.

For further investigating the sensing performance of the integrated structure, we change the insulator of air to be the one with the index range from $1.33-$ 1.36. Certainly, the $Q$ factor, sensitivity $S$, detection limit (DL), and the figure of merit (FOM) are the significant factors of sensors to evaluate the performances, and they are defined as $[7,42,43]$

$$
Q=\frac{\lambda_{r}}{\text { FWHM }}
$$

$$
\begin{gathered}
S=\frac{\partial \lambda_{r}}{\partial n} \\
\mathrm{DL}=\frac{\mathrm{FWHM}}{S} \\
\mathrm{FOM}=\frac{S}{\text { FWHM }}
\end{gathered}
$$

where $\lambda_{r}$ is the central wavelength, and $T\left(\lambda_{r}\right)$ is the transmittance corresponding to the dip wavelength. It should be noted that the detection limit is defined as the minimal detectable refractive index change caused by the analyte. In an actual sensing event, the smallest detectable shift of the resonant wavelength depends on the FWHM of the transmission dip, so DL is defined in (9). After setting $n=1.33,1.34,1.35$, and 1.36, the transmission spectra based on FDTD simulations are shown in Fig. 9. Obviously, there are redshifts for all the dips by increasing the index, and four channels of M1, M2, M3, and B are obtained at the wavelength range of $1000 \mathrm{~nm}-1800 \mathrm{~nm}$. All the performances, including $Q, S, \mathrm{DL}$, and FOM, are shown in Table 1. Obviously, the sensitivity of the dip at the short wavelength is lower than that of the dip at the long wavelength. However, the $Q$ factor and DL almost show the opposite trend. The optimal values of $Q, S$, and DL are $110,1500 \mathrm{~nm} / \mathrm{RIU}$, and 0.0125 , respectively. Especially for the sensitivity, it 


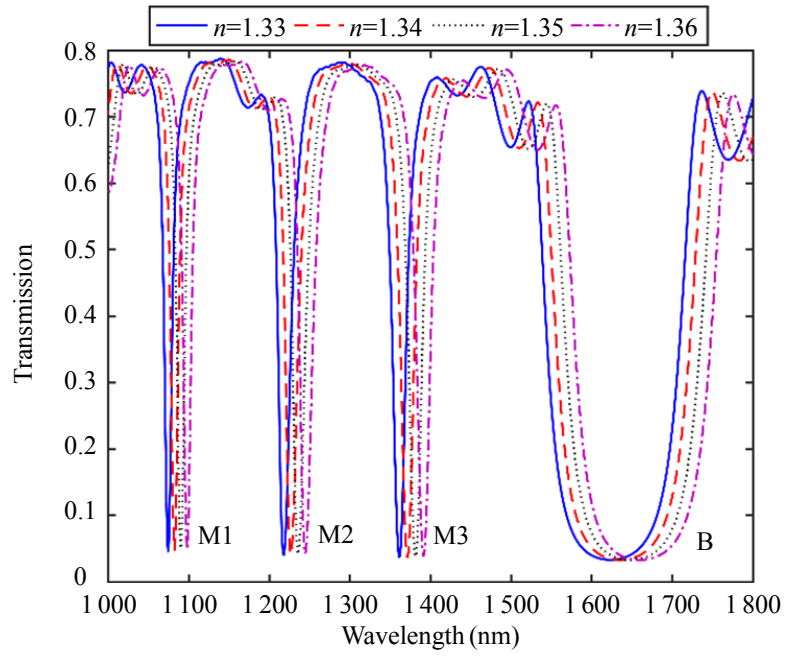

Fig. 9 Transmission spectra with RI of $1.33,1.34,1.35$, and 1.36 , respectively.

Table $1 \lambda, Q, S, \mathrm{DL}$, and FOM of the dips by defining $n=1.33,1.34,1.35$, and 1.36 , respectively.

\begin{tabular}{|c|c|c|c|c|c|}
\hline \multicolumn{2}{|c|}{$\begin{array}{l}\text { Refractive index } \\
\text { Channels }\end{array}$} & 1.33 & 1.34 & 1.35 & 1.36 \\
\hline \multirow{5}{*}{ M1 } & $\lambda(\mathrm{nm})$ & 1074 & 1082 & 1090 & 1098 \\
\hline & $Q$ & 107 & 108 & 109 & 110 \\
\hline & $S(\mathrm{~nm} / \mathrm{RIU})$ & \multicolumn{4}{|c|}{800} \\
\hline & DL & \multicolumn{4}{|c|}{0.0125} \\
\hline & FOM & \multicolumn{4}{|c|}{80.0} \\
\hline \multirow{5}{*}{ M2 } & $\lambda(\mathrm{nm})$ & 1217 & 1227 & 1236 & 1244 \\
\hline & $Q$ & 68 & 68 & 69 & 69 \\
\hline & $S(\mathrm{~nm} / \mathrm{RIU})$ & \multicolumn{4}{|c|}{900} \\
\hline & DL & \multicolumn{4}{|c|}{0.0178} \\
\hline & FOM & \multicolumn{4}{|c|}{56.2} \\
\hline \multirow{5}{*}{ M3 } & $\lambda(\mathrm{nm})$ & 1361 & 1371 & 1382 & 1391 \\
\hline & $Q$ & 76 & 76 & 73 & 73 \\
\hline & $S(\mathrm{~nm} / \mathrm{RIU})$ & \multicolumn{4}{|c|}{1000} \\
\hline & DL & \multicolumn{4}{|c|}{0.0180} \\
\hline & FOM & \multicolumn{4}{|c|}{55.6} \\
\hline \multirow{5}{*}{ B } & $\lambda(\mathrm{nm})$ & 1620 & 1636 & 1649 & 1665 \\
\hline & $Q$ & 10 & 10 & 10 & 10 \\
\hline & $S(\mathrm{~nm} / \mathrm{RIU})$ & \multicolumn{4}{|c|}{1500} \\
\hline & DL & \multicolumn{4}{|c|}{0.1087} \\
\hline & FOM & \multicolumn{4}{|c|}{9.2} \\
\hline
\end{tabular}

is higher than the recently reported results, i.e., $1477 \mathrm{~nm} / \mathrm{RIU}$ in [7], $1295 \mathrm{~nm} / \mathrm{RIU}$ in [44], and $1120 \mathrm{~nm} / \mathrm{RIU}$ in [45]. The values of the FOM for channels M1, M2, M3, and B are 80.0, 56.2, 55.6, and 9.2, respectively. Obviously, the FOMs of the MZI channels are much larger than that of the Bragg reflection channel. But it should be noted that the highest sensitivity of $1500 \mathrm{~nm} / \mathrm{RIU}$ is obtained from the Bragg reflector spectrum which has a larger FWHM but the low FOM and $Q$ factor. Thus, it may be not suitable for index sensing but is suitable for broadband filtering. However, the MZI channels possessing higher FOMs and $Q$ factors could be employed in the sensing area even the sensitivities are a little lower. Consequently, we believe that the proposed structure can be applied in the index sensing area, and the symmetrical line shapes for all channels are also suitable for the optical filter.

\section{Conclusions}

In summary, an integrated-plasmonic chip of the Bragg grating and MZI has been proposed and investigated by using MIM waveguides. When the metallic gratings are placed in the center of an MIM waveguide, the Bragg reflection with a large bandwidth is achieved at $1550 \mathrm{~nm}$ by setting the metallic grating length and period as $240 \mathrm{~nm}$ and $645 \mathrm{~nm}$, respectively. Besides, the MZI effect is also obtained by shifting the grating $10 \mathrm{~nm}$ away from the center of the waveguide, since the asymmetrical waveguide led to different indices and phase delays. The wavelengths of the Bragg reflector and the MZI could be manipulated by the period and the grating length, respectively. Furthermore, three MZI channels and one Bragg reflection channel have been achieved after cascading three different gratings in a large period. In the index range of 1.33 - 1.36, the sensitivities for all the dips have also been calculated as $800 \mathrm{~nm} / \mathrm{RIU}, 900 \mathrm{~nm} / \mathrm{RIU}$, $1000 \mathrm{~nm} / \mathrm{RIU}$, and $1500 \mathrm{~nm} / \mathrm{RIU}$, respectively. Consequently, this proposed structure could serve as highly integrated optical devices, such as chip-scale sensors, filters, and modulators.

\section{Acknowledgment}

This work was supported by the National Key Research and Development Program of China (Grant No. 2019YFB1803505), the National Natural Science Foundation of China (Grant Nos. U2001601, 62175039, and 61925501), the Science and 
Technology Project of Guangzhou (Grant No. 201904010243), Major Special Projects in Guangdong Province (Grant No. 2018B010114002), the Engineering Research Center of Digital Imaging and Display, Ministry of Education, Soochow University (Grant No. SDGC2133), and the Program for Guangdong Introducing Innovative and Enterpreneurial Teams.

Open Access This article is distributed under the terms of the Creative Commons Attribution 4.0 International License (http://creativecommons.org/licenses/by/4.0/), which permits unrestricted use, distribution, and reproduction in any medium, provided you give appropriate credit to the original author(s) and the source, provide a link to the Creative Commons license, and indicate if changes were made.

\section{References}

[1] W. L. Barnes, A. Dereux, and T. W. Ebbesen, "Surface plasmon subwavelength optics," Nature, 2003, 424(6950): 824-830.

[2] X. M. Wen, Y. G. Bi, F. S. Yi, X. L. Zhang, Y. F. Liu, W. Q. Wang, et al., "Tunable surface plasmon-polariton resonance in organic light-emitting devices based on corrugated alloy electrodes," Opto-Electronic Advances, 2021, 4(8): 200024-1-200024-7.

[3] R. Zafar, S. Nawaz, G. Singh, A. D'Alessandro, and M. Salim, "Plasmonics-based refractive index sensor for detection of hemoglobin concentration," IEEE Sensors Journal, 2018, 18(11): 4372-4377.

[4] Z. Li, W. W. Liu, Z. C. Li, H. Cheng, S. Q. Chen, and J. G. Tian, "Fano-resonance-based mode-matching hybrid metasurface for enhanced second-harmonic generation," Optics Letters, 2017, 42(16): 3117-3120.

[5] L. W. Zhang, Q. Wang, and W. W. Meng, "Dual-band absorption enhancement of monolayer transition-metal dichalcogenides in metamaterials," Optoelectronics Letters, 2021, 17(7): 412-417.

[6] Z. Guo, K. Wen, Y. Qin, Y. Fang, Z. Li, and L. Chen, "A plasmonic refractive-index sensor based multiple Fano resonance multiplexing in slot-cavity resonant system," Photonic Sensors, 2022, 12(2): 175-184.

[7] S. L. Li, Y. L. Wang, R. Z. Jiao, L. L. Wang, G. Y. Duan, and L. $\mathrm{Yu}$, "Fano resonances based on multimode and degenerate mode interference in plasmonic resonator system," Optics Express, 2017, 25(4): 3525-3533.

[8] Z. Chen, J. J. Chen, L. Yu, and J. H. Xiao, "Sharp trapped resonances by exciting the anti-symmetric waveguide mode in a metal-insulator-metal resonator," Plasmonics, 2015, 10(1): 131-137.

[9] G. C. Wang, A. Shen, C. Y. Zhao, L. Z. Yang, T. G. Dai, Y. H. Wang, et al., "Fano-resonance-based ultra-high-resolution ratio-metric wavelength monitor on silicon," Optics Letters, 2016, 41(3): 544-547.

[10] L. Chen, L. Zhang, and X. Xu, "Tuning of the graphene surface plasmon by the monolayer $\mathrm{MoS}_{2}$," Optoelectronics Letters, 2021, 17(11): 646-650.

[11] Q. Chen, L. Liang, Q. L. Zheng, Y. X. Zhang, and L. Wen, "On-chip readout plasmonic mid-IR gas sensor," Opto-Electronic Advances, 2020, 3(7): 07190040.

[12] G. T. Cao, H. J. Li, Y. Deng, S. P. Zhan, Z. H. He, and B. X. Li, "Plasmon-induced transparency in a single multimode stub resonator," Optics Express, 2014, 22(21): 25215-25223.

[13] Y. K. Gong, X. M. Liu, and L. R. Wang, "High-channel-count plasmonic filter with the metal-insulator-metal Fibonacci-sequence gratings," Optics Letters, 2010, 35(3): 285-287.

[14] X. Luo, X. H. Zou, X. F. Li, Z. Zhou, W. Pan, L. S. Yan, et al., "High-uniformity multichannel plasmonic filter using linearly lengthened insulators in metal-insulator-metal waveguide," Optics Letters, 2013, 38(9): 1585-1587.

[15] R. E. Holmlin, R. Haag, M. L. Chabinyc, R. F. Ismagilov, A. E. Cohen, A. Terfort, et al., "Electron transport through thin organic films in metal-insulator-metal junctions based on self-assembled monolayers," Journal of the American Chemical Society, 2001, 123(21): 5075-5085.

[16] F. Galvez, J. del Valle, A. Gomez, M. R. Osorio, D. Granados, D. Pérez de Lara, et al., "Plasmonic nanodevice with magnetic funcionalities: fabrication and characterization," Optical Materials Express, 2016, 6(10): 3086-3096.

[17] S. P. Zhan, H. J. Li, G. T. Cao, Z. H. He, B. X. Li, and H. Yang, "Slow light based on plasmon-induced transparency in dual-ring resonator-coupled MDM waveguide system," Journal of Physics D: Applied Physics, 2014, 47(20): 205101.

[18] K. Wen, L. Chen, J. Zhou, L. Lei, and Y. Fang, "A plasmonic chip-scale refractive index sensor design based on multiple Fano resonances," Sensors, 2018, 18(10): 3181 .

[19] M. A. Butt, S. N. Khonina, and N. L. Kazanskiy, "Plasmonics: a necessity in the field of sensing-a review (invited)," Fiber and Integrated Optics, 2021, 40(1): 14-47.

[20] Q. He, Y. P. Huo, Y. Y. Guo, Q. Q. Niu, X. X. Hao, P. F. Cui, et al., "Multiple adjustable Fano resonance based on double half ring resonator and its application," Physica Scripta, 2021, 96(6): 065504.

[21] C. Chao, Y. Chau, and H. P. Chiang, "Highly sensitive metal-insulator-metal plasmonic refractive 
index sensor with a centrally coupled nanoring containing defects," Journal of Physics D: Applied Physics, 2021, 54(11): 115301.

[22] S. M. Shen, S. C. She, Z. Y. Wang, Q. L. Tan, J. J. Xiong, and W. D. Zhang, "MIM waveguide structure consisting of two triangle stubs, side-coupled with an eight-like resonant cavity," Optics Communications, 2021, 495: 127087.

[23] M. A. Butt, A. Kazmierczak, N. L. Kazanskiy, and S. N. Khonina, "Metal-insulator-metal waveguide-based racetrack integrated circular cavity for refractive index sensing application," Electronics, 2021, 10(12): 1419.

[24] X. X. Hao, Y. P. Huo, Q. He, Y. Y. Guo, Q. Q. Niu, P. F. Cui, et al., "Multiple plasmon-induced transparency with extra-high FOM based on a MIM waveguide composed of stubs," Physica Scripta, 2021, 96(7): 075505 .

[25] R. Al Mahmud, M. O. Faruque, and R. H. Sagor, "Plasmonic refractive index sensor based on ring-type pentagonal resonator with high sensitivity," Plasmonics, 2021, 16(3): 873-880.

[26] H. R. Shi, S. B. Yan, X. Y. Yang, X. S. Wu, W. C. $\mathrm{Wu}$, and E. T. Hua, "A nanosensor based on a metal-insulator-metal bus waveguide with a stub coupled with a racetrack ring resonator," Micromachines, 2021, 12(5): 495.

[27] S. N. Khonina, N. L. Kazanskiy, M. A. Butt, A. Kazmierczak, and R. Piramidowicz, "Plasmonic sensor based on metal-insulator-metal waveguide square ring cavity filled with functional material for the detection of $\mathrm{CO}_{2}$ gas," Optics Express, 2021, 29(11): 16584-16594.

[28] Z. J. Hu, R. Ma, X. J. Zhang, Z. Y. Sun, X. Liu, J. Liu, et al., "Weak feedback assisted random fiber laser from $45^{\circ}$-tilted fiber Bragg grating," Optics Express, 2019, 27(3): 3255-3263.

[29] D. Zheng, Z. Cai, I. Floris, J. Madrigal, W. Pan, X. H. Zou, et al., "Temperature-insensitive optical tilt sensor based on a single eccentric-core fiber Bragg grating," Optics Letters, 2019, 44(22): 5570-5573.

[30] V. Ahsani, F. Ahmed, M. B. G. Jun, and C Bradley, "Tapered fiber-optic Mach-Zehnder interferometer for ultra-high sensitivity measurement of refractive index," Sensors, 2019, 19(7): 1652.

[31] S. Kumar, Z. Guo, R. Singh, Q. L. Wang, B. Y. Zhang, S. Cheng, et al., " $\mathrm{MoS}_{2}$ functionalized multicore fiber probes for selective detection of shigella bacteria based on localized plasmon," Journal of Lightwave Technology, 2021, 39(12): 4069-4081.

[32] M. Lobry, M. Loyez, K. Chah, E. M. Hassan, E. Goormaghtigh, M. C. DeRosa, et al., "HER2 biosensing through spr-envelope tracking in plasmonic optical fiber gratings," Biomedical Optics Express, 2020, 11(9): 4862-4871.
[33] C. Leitão, A. Leal-Junior, A. R. Almeida, S. O. Pereira, F. M. Costa, J. L. Pinto, et al., "Cortisol AuPd plasmonic unclad POF biosensor," Biotechnology Reports, 2021, 29: e00587.

[34] R. Guo, M. Decker, F. Setzpfandt, I. Staude, D. N. Neshev, and Y. S. Kivshar, "Plasmonic Fano nanoantennas for on-chip separation of wavelengthencoded optical signals," Nano Letters, 2015, 15(5): 3324-3328.

[35] M. F. Limonov, M. V. Rybin, A. N. Poddubny, and Y. S. Kivshar, "Fano resonances in photonics," Nature Photonics, 2017, 11(9): 543-554.

[36] J. A. Dionne, L. A. Sweatlock, and H. A. Atwater, "Plasmon slot waveguides: towards chip-scale propagation with subwavelength-scale localization," Physical Review B, 2006, 73(3): 035407.

[37] P. B. Johnson and R. W. Christy, "Optical constants of the noble metals," Physical review B, 1972, 6(12): 4370-4379.

[38] N. Lin, L. Langguth, T. Weiss, J. Kastel, M. Fleischhauer, T. Pfau, et al., "Plasmonic analogue of electromagnetically induced transparency at the Drude damping limit," Nature Materials, 2009, 8(9): 758-762.

[39] D. Wei, "The study on the structure, plasmonic resonance modes and SERS properties of metallic nanoparticle arrays fabricated by nanosphere lithography," Ph.D. dissertation, Nanjing University, Nanjing, 2017.

[40] H. T. Miyazaki and Y. Kurokawa, "Squeezing visible light waves into a 3-nm-thick and 55-nm-long plasmon cavity," Physical Review Letters, 2006, 96(9): 097401.

[41] F. Ye, M. J. Burns, and M. J. Naughton, "Symmetrybroken metamaterial absorbers as reflectionless directional couplers for surface plasmon polaritons in the visible range," Advanced Optical Materials, 2014, 2(10): 957-965.

[42] C. Y. Chao and L. Guo, "Design and optimization of microring resonators in biochemical sensing applications," Journal of Lightwave Technology, 2006, 24(3): 1395-1402.

[43] J. L. Zhang, L. Shi, S. Zhu, X. B. Xu, and X. L. Zhang, "Modeling of a single-notch microfiber coupler for high-sensitivity and low detection-limit refractive index sensing," Sensors, 2016, 16(5): 672.

[44] M. Rahmatiyar, M. Afsahi, and M. Danaie, "Design of a refractive index plasmonic sensor based on a ring resonator coupled to a MIM waveguide containing tapered defects," Plasmonics, 2020, 15(6): 2169-2176.

[45] Y. Chen, Y. Xu, and J. Cao, "Fano resonance sensing characteristics of MIM waveguide coupled square convex ring resonator with metallic baffle," Results in Physics, 2019, 14: 102420. 